\title{
Resolution Improvement in Stage-Scanning Electron Holography: Comparison with Conventional Electron Holography
}

\author{
Dan Lei, ${ }^{1}$ Kazutaka Mitsuishi, ${ }^{2}$ Ken Harada, ${ }^{3}$ Masayuki Shimojo, ${ }^{4}$ \\ Dongying Ju, ${ }^{1}$ and Masaki Takeguchi ${ }^{5}$ \\ ${ }^{1}$ Department of Materials Science and Engineering, Saitama Institute of Technology, 1690 Fusaiji, Fukaya, \\ Saitama 369-0293, Japan \\ ${ }^{2}$ Surface Physics and Structure Unit, National Institute for Materials Science, 3-13 Sakura, Tsukuba 305-0003, Japan \\ ${ }^{3}$ Advanced Measurement and Analysis Center, Central Research Laboratory, Hitachi Ltd., Hatoyama, Saitama 350-0395, Japan \\ ${ }^{4}$ Department of Materials Science and Engineering, Shibaura Institute of Technology, 3-7-5 Toyosu, Koto-ku, \\ Tokyo 135-8548, Japan \\ ${ }^{5}$ Transmission Electron Microscopy Station, National Institute for Materials Science, 3-13 Sakura, Tsukuba 305-0003, Japan
}

Correspondence should be addressed to Dan Lei; lei.dan@nims.go.jp

Received 13 May 2013; Accepted 26 June 2013

Academic Editors: I.-C. Chen, C. Malagù, and B. Pignataro

Copyright (C) 2013 Dan Lei et al. This is an open access article distributed under the Creative Commons Attribution License, which permits unrestricted use, distribution, and reproduction in any medium, provided the original work is properly cited.

\begin{abstract}
Electron holography provides information on the phase and amplitude of electron wave passing through a specimen. The recently proposed stage-scanning electron holography technique should improve the spatial resolution of phase and amplitude images compared to the conventional electron holography based on the Fourier transformation method. To demonstrate the resolution improvement, cobalt nanoparticles were observed using the stage-scanning holography and the conventional holography, and significantly sharper images were obtained with the former technique.
\end{abstract}

\section{Introduction}

Electron holography [1-3] is a transmission electron microscopy (TEM) technique that uses a biprism to mix an object wave passing through a specimen with the reference wave passing through vacuum. Interaction of the two waves produces a pattern containing interference fringes. In contrast to conventional TEM techniques, which only record the spatial distribution of image intensity, electron holography yields information on both the phase and amplitude of the object wave through reconstruction of the interference pattern, or hologram. The phase distribution can then be used to provide information about the magnetic and electrostatic fields in the specimen.

To obtain information on the phase and amplitude, a reconstruction process is necessary such as the Fourier transformation method $[4,5]$, in which the electron hologram is Fourier-transformed, and then its selected sideband is inversely Fourier-transformed. In this approach, the spatial resolution of the reconstructed phase image is limited by the fringe spacing in the hologram [6-10]. This spacing determines the separation of center band and side band in Fourier space and thus the resolution of resultant phase and amplitude images. However, the use of small fringe spacing, aiming for higher resolution, results in a lower fringe contrast and signal-to-noise ratio. Many efforts have been spent to overcome this difficulty. For example, $\mathrm{Ru}$ et al. $[6,7]$ developed a phase-shifting electron holography technique, where the incident beam is tilted to obtain a series of holograms with different initial phases. With this method, the object wave passing through the specimen can be determined independently of the fringe spacing without requiring Fourier transformation.

We presented an electron holography technique using a stage-scanning system [11]. In this method, line intensities are acquired from a series of holograms recorded at different 


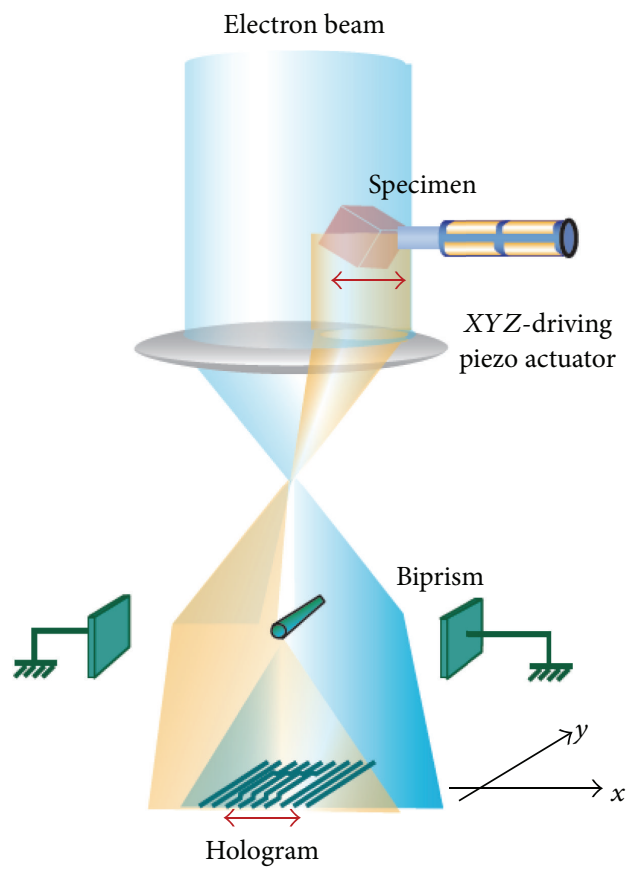

FIGURE 1: Schematic of electron holography using a stage-scanning specimen holder.

specimen positions, and an interferogram that corresponds to the phase distribution can be obtained directly, without complicated reconstruction such as Fourier transformation. We further improved this method by recording a series of holograms as a 3-dimensional (3D) data cube at different specimen positions [12]. Slicing the 3D data cube at different CCD pixels produces several interferograms. By applying the proposed reconstruction procedure to these interferograms, the phase distribution can be reconstructed with high precision. This technique is expected to overcome the limitation of spatial resolution due to the fringe spacing.

In this paper, we demonstrate the resolution enhancement by observing Co nanoparticles with the stage-scanning electron holography and compare the results with the conventional holography based on the Fourier transformation method. We used a low-magnification mode in which the objective lens of the microscope is switched off. To realize the electron holography configuration, the first intermediate lens is also turned off, which limits the choice of magnification. Therefore, it may be difficult to have an appropriate combination of magnification and fringe spacing for performing conventional electron holography on specimens with micrometer-scale features. The stage-scanning electron holography technique [12] should solve this problem by overcoming the limitation between the spatial resolution and fringe spacing. This flexibility is especially important for observing magnetic specimens, which must be located in a low-field region to avoid unwanted magnetic saturation.

\section{Experimental Methods}

Figure 1 shows the electron optics and instruments used in the stage-scanning holography. To acquire an electron hologram, the region of interest on the specimen should be positioned to cover half the field of view. An electron wave is divided into two: an object wave modulated by the specimen and a reference wave passing through vacuum. Application of voltage to an electrostatic biprism located below the specimen results in an overlap of the reference wave and the object wave and in the appearance of interference fringes. The fringe spacing and the width of the interference region are determined by the biprism voltage. A stage-scanning system [13-15], which comprises a specially designed holder equipped with a piezo-driven specimen stage, a power supply, and control software, was used in this experiment. Using this stage-scanning system, the specimen is moved in a fixed electron-optics configuration, and a series of holograms are recorded at different specimen positions.

The object wave is then reconstructed from the recorded series of holograms. The details of the reconstruction procedure are given in our previous paper [12] and are briefly repeated here.

When the biprism is oriented along the $y$ direction, the electron hologram is obtained as a result of the interference between the object wave $\Phi_{o}$ and reference wave $\Phi_{r}$ as

$$
\begin{aligned}
I(n, x, y)= & \left|\Phi_{o}+\Phi_{r}\right|^{2} \\
= & \left|\phi_{0}(x-n \Delta x, y)\right|^{2}+1 \\
& +2 \phi_{0}(x-n \Delta x, y) \\
& \quad \times \cos \left[\eta(x-n \Delta x, y)+2 \pi \frac{x}{m}\right] .
\end{aligned}
$$

Here, $x$ and $y$ denote the position in a hologram, $\phi_{0}$ and $\eta$ refer to the amplitude and phase, respectively, $\Delta x$ is the scan step along the $x$ direction, $n$ is the index of scan steps, and $m$ refers to the fringe spacing. The recorded series of holograms with different specimen positions can be viewed as a $3 \mathrm{D}$ data cube with the dimensions $(x, y, \Delta x \cdot n)$.

The reconstruction procedure of the stage-scanning holography consists of three steps: formation of interferograms by slicing the $3 \mathrm{D}$ data cube at different $x$ positions, alignment of the specimen positions on the interferograms, and reconstruction of the object wave.

Slicing the cube in the $(y, \Delta x \cdot n)$ plane at $x=x_{k}$ extracts an interferogram

$$
\begin{aligned}
\Pi_{k}(n, y)= & \left|\phi_{0}\left(x_{k}-n \Delta x, y\right)\right|^{2}+1 \\
+ & 2 \phi_{0}\left(x_{k}-n \Delta x, y\right) \\
& \quad \times \cos \left[\eta\left(x_{k}-n \Delta x, y\right)+2 \pi \frac{x_{k}}{m}\right],
\end{aligned}
$$

where the fringe spacing $m$ is set to be an integer and a multiple of CCD pixel sizes and is divided by $N$. Thus $x_{k}$ can be expressed as $x_{k}=(m / N) k,(k=0,1,2 \ldots, N-1)$. 
The specimen positions are offset on the interferograms with different $x_{k}$ and are aligned using the following equation:

$$
\begin{aligned}
\Pi_{k}^{\prime}(n, y)= & \Pi_{k}\left(\left(n+\frac{m}{N \Delta x} k\right), y\right) \\
= & \left|\phi_{0}(-n \Delta x, y)\right|^{2}+1 \\
& +2 \phi_{0}(-n \Delta x, y) \cos \left[\eta(-n \Delta x, y)+2 \pi \frac{k}{N}\right]
\end{aligned}
$$

The interferogram $\Pi_{k}^{\prime}(n, y)$ is the one after alignment.

Multiplying (3) by $\exp (-2 \pi i(k / N))$ and summing both sides over $k$ yields

$$
\begin{aligned}
\sum_{k=0}^{N-1} \Pi_{k}^{\prime}(n, y) \exp \left(-2 \pi i \frac{k}{N}\right) & \\
= & N \phi_{0}(-n \Delta x, y) \cos [\eta(-n \Delta x, y)] \\
& +i N \phi_{0}(-n \Delta x, y) \sin [\eta(-n \Delta x, y)]
\end{aligned}
$$

Then, the phase image and amplitude image can be obtained, respectively, as

$$
\begin{gathered}
\eta(-n \Delta x, y)=\tan ^{-1}\left(\frac{\mathrm{Im}}{\mathrm{Re}}\right), \\
\phi_{0}(-n \Delta x, y)=\frac{1}{N} \sqrt{(\operatorname{Im})^{2}+(\mathrm{Re})^{2}}
\end{gathered}
$$

where Im and Re denote the imaginary and real parts of the term $\sum_{k=0}^{N-1} \Pi_{k}^{\prime}(n, y) \exp (-2 \pi i(k / N))$ in (4), respectively.

An important improvement of this technique is that the spatial resolution of the reconstructed phase image is determined not by the interference fringe spacing, but by the scan step and by the microscope resolution or the pixel size along the $y$ direction. This is the principal difference from the conventional holography as mentioned in the introduction. Because the Fourier transformation method is unnecessary to reconstruct the phase, coarse fringes with high contrast can be used, which would also be helpful for improving the precision of the reconstructed phase image.

Experiments were carried out in the low-magnification mode with a JEOL JEM-ARM200F microscope $(200 \mathrm{kV})$ equipped with a biprism and a stage-scanning system. Co nanoparticles with a diameter of $10-20 \mathrm{~nm}$ deposited on a carbon film were used as a sample.

\section{Results and Discussion}

Figure 2 shows a bright-field TEM image of Co particles dispersed on the edge of a carbon film. Holograms were acquired at different specimen positions, by moving the specimen with the stage-scanning system, and saved as a $3 \mathrm{D}$ data cube with dimensions of 155 pixels $\times 260$ pixels $\times$ 90 steps. The total acquisition time was about $1 \mathrm{~min} 40 \mathrm{~s}$, including data transfer and 1 second exposure for each scan step. Two holograms, recorded at different specimen

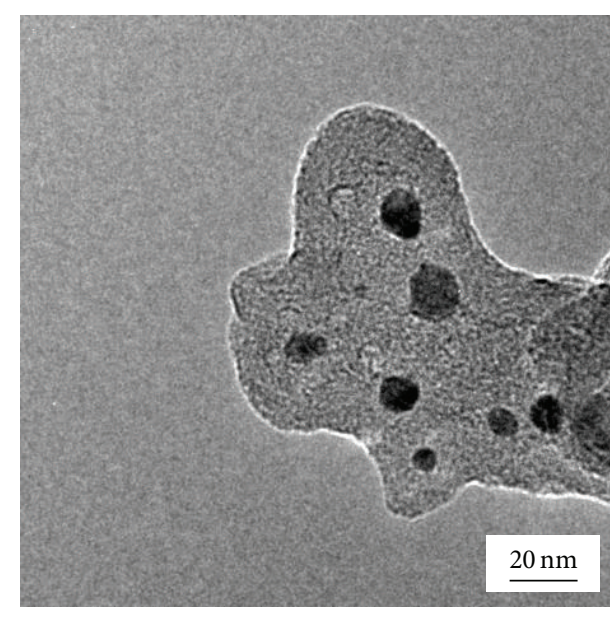

FIgURE 2: TEM image of Co particles.

positions and extracted from the 3D data cube, are shown in Figures 3(a) and 3(b). In this case, the fringe spacing was $26 \mathrm{~nm}$ or 10 pixels, which is wider than the diameter of the particles. In the extracted holograms (a) and (b), the particles cannot be distinguished, but only the fringe shift on the specimen can be seen. We reconstructed the phase distribution of the particles (Figure 3(c)) using 10 lines of CCD pixels as a fringe, 90 holograms, and stage-scanning distance of $230 \mathrm{~nm}$. The Co particles are well distinguished in Figure 3(c).

Figure 4 shows the line profile across a Co particle indicated by the white line in Figure 3(c). The profile shows the shape of the particle with a phase change of about 1.2 radians and a diameter of about $15.5 \mathrm{~nm}$, which is close to $14.1 \mathrm{~nm}$ measured from the TEM image of Figure 2. Comparing with the results of the conventional holography shown next, the proposed technique yields higher precision in measuring the particle size. The phase change on both sides of the particle is due to the thickness variation of the carbon film.

For comparison, the same Co particles were observed with the conventional holography technique based on the Fourier transformation method. In this case, fine fringe spacing was necessary to attain a high resolution. Otherwise, windowing the sideband in the Fourier space might induce artifacts in the real space [16]. Therefore, a fringe spacing of $17 \mathrm{~nm}$ was used in the same optical configuration with a higher biprism voltage, which was the finest fringe spacing considering the fringe contrast and signal-to-noise ratio of the holograms given the limited freedom of magnification in this experiment. Figures 5(a) and 5(b) show the hologram and the phase distribution of the Co particles retrieved via Fourier transformation, respectively. It is difficult to distinguish the phase of each Co particle in this reconstructed phase image because of the low spatial resolution. In Fourier transformation method, the size of the selected sideband region should be small enough for avoiding the mixture of the sideband and the center band. This requirement limits the number of pixels of the reconstructed image and reduces the spatial resolution of the reconstructed image. Comparing the 


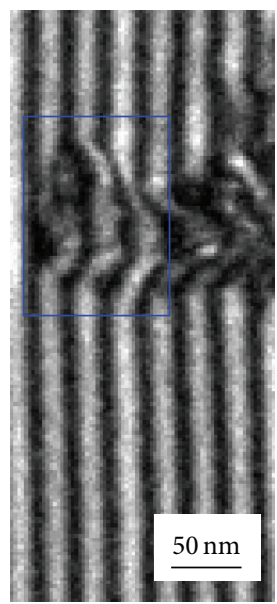

(a)

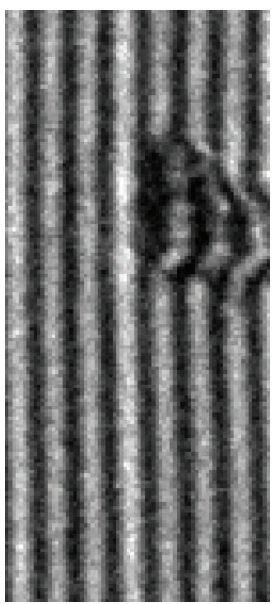

(b)

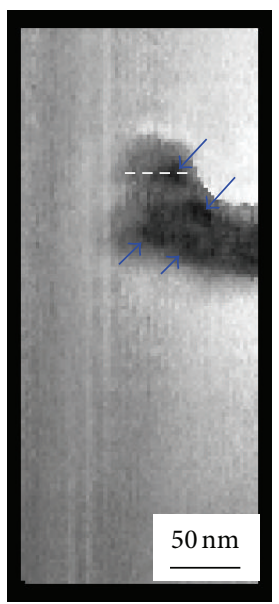

(c)

Figure 3: Two extracted holograms of the Co particles (a) and (b) with a fringe spacing of $26 \mathrm{~nm}$. The specimen moved from the position in hologram (a) to the position in hologram (b) due to the movement of the specimen stage. (c) The phase image obtained with the stagescanning technique.

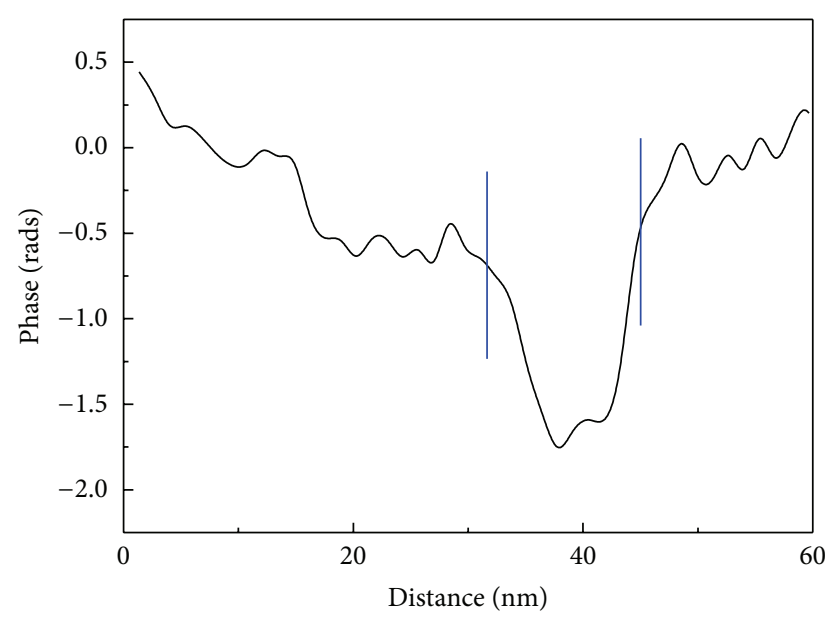

Figure 4: Profile of phase change of the Co particle indicated by the white line in Figure 3(c).

results from these two techniques, we conclude that the stagescanning holography yields higher resolution with a wider fringe spacing than the conventional holography based on the Fourier transformation method. The former technique is useful when fine fringes cannot be obtained but a high resolution is needed.

The major difference between the stage-scanning holography and conventional holography based on Fourier transformation is the necessity of fine fringe spacing. In the conventional holography, the fringe spacing should be about three times finer than the desired spatial resolution [610]. Thus, many studies focused on decreasing the fringe spacing, although this will also decrease the fringe contrast. In our stage-scanning holography, the spatial resolution is determined by the scan step width but not the fringe spacing. Therefore, we can use a wide fringe spacing for reconstruction and then obtain a high-contrast interference pattern. Also, in the conventional electron holography method, if the specimen has sharp edges or large phase variations, the Fourier spectrum of the specimen will extend widely, hampering the separation of the sidebands from the central band. Imperfect separation will distort the overall area of the reconstructed image. The stage-scanning holography does not suffer from this problem.

In practice, several factors can introduce artifacts into the reconstructed phase image. For example, the drift of the specimen and/or of the biprism may introduce phase errors or distortions to the specimen shape. These effects can hopefully be reduced in the future by improving the biprism stage and the specimen stage. Moreover, the stage-scanning system is driven by a piezo, which results in inconsistent steps due to hysteresis effects and in the concomitant errors in the phase calculation.

\section{Summary}

The stage-scanning electron holography allows retrieving phase distribution without Fourier transformation. As a result, the spatial resolution can be determined independently of the fringe spacing of the holograms. In this study, we applied the stage-scanning electron holography in a lowmagnification mode to Co particles. Higher spatial resolution was achieved compared with that of conventional electron holography based on the Fourier transformation method. The stage-scanning electron holography is thus useful in a lowmagnification mode when fine fringes cannot be obtained due to the limited TEM magnification.

\section{Acknowledgments}

The authors are grateful to Dr. Iakoubovskii for proofreading the paper. A part of this study was financially supported by the Budgets for "Development of Environmental Technologies 


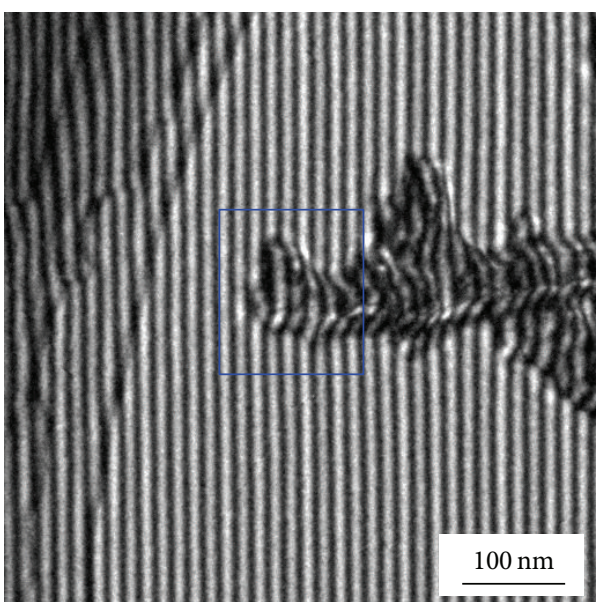

(a)

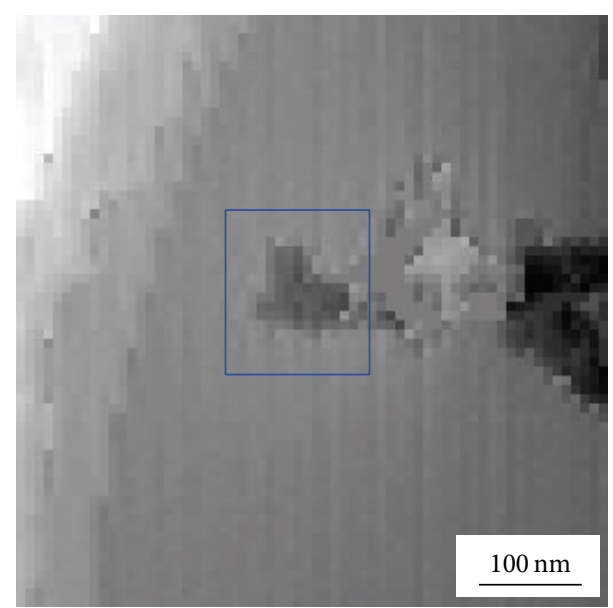

(b)

FIGURE 5: Hologram taken with a fine fringe spacing of $17 \mathrm{~nm}$ by conventional electron holography (a) and the reconstructed phase image (b). The area within the blue box is the same area that was observed by the stage-scanning holography.

Utilizing Nanotechnology" and "the Low-Carbon Research Network in Japan (LCnet)" of the Ministry of Education, Culture, Sports, Science and Technology.

\section{References}

[1] D. Gabor, "Microscopy by reconstructed wave-fronts," Proceedings of the Royal Society London, vol. 197, pp. 454-487, 1949.

[2] H. Lichte and M. Lehmann, "Electron holography-basics and applications," Reports on Progress in Physics, vol. 71, no. 1, Article ID 016102, 2008.

[3] A. Tonomura, Electron Holography, Springer, Berlin, Germany, 1993.

[4] M. Takeda, H. Ina, and S. Kobayashi, "Fourier-transform method of fringe-pattern analysis for computer-based tomography and interferometry," Journal of the Optical Society of America, vol. 72, no. 1, pp. 156-160, 1982.

[5] T. Fujita, K. Yamamoto, M. R. McCartney, and D. J. Smith, "Reconstruction technique for off-axis electron holography using coarse fringes," Ultramicroscopy, vol. 106, no. 6, pp. 486491, 2006

[6] Q. Ru, J. Endo, T. Tanji, and A. Tonomura, "Phase-shifting electron holography by beam tilting," Applied Physics Letters, vol. 59, no. 19, pp. 2372-2374, 1991.

[7] G. Lai, Q. Ru, K. Aoyama, and A. Tonomura, "Electronwave phase-shifting interferometry in transmission electron microscopy," Journal of Applied Physics, vol. 76, no. 1, pp. 39-45, 1994.

[8] W. J. De Ruijter and J. K. Weiss, "Detection limits in quantitative off-axis electron holography," Ultramicroscopy, vol. 50, no. 3, pp. 269-283, 1993.

[9] K. Yamamoto, T. Hirayama, and T. Tanji, "Off-axis electron holography without Fresnel fringes," Ultramicroscopy, vol. 101, no. 2-4, pp. 265-269, 2004.

[10] H. Lichte, "Performance limits of electron holography," Ultramicroscopy, vol. 108, no. 3, pp. 256-262, 2008.

[11] D. Lei, K. Mitsuishi, K. Harada, M. Shimojo, D. Y. Ju, and M. Takeguchi, "Mapping of phase distribution in electron holography with a stage-scanning system," Materials Science Forum, vol. 750, pp. 152-155, 2013.

[12] D. Lei, K. Mitsuishi, K. Harada, M. Shimojo, D. Y. Ju, and M. Takeguchi, "Direct acquisition of interferogram by stage scanning in electron interferometry," Microscopy, 2013.

[13] M. Takeguchi, M. Shimojo, M. Tanaka, R. Che, W. Zhang, and K. Furuya, "Electron holographic study of the effect of contact resistance of connected nanowires on resistivity measurement," Surface and Interface Analysis, vol. 38, no. 12-13, pp. 1628-1631, 2006.

[14] M. Takeguchi, A. Hashimoto, M. Shimojo, K. Mitsuishi, and K. Furuya, "Development of a stage-scanning system for highresolution confocal STEM," Journal of Electron Microscopy, vol. 57, no. 4, pp. 123-127, 2008.

[15] M. Takeguchi, K. Mitsuishi, D. Lei, and M. Shimojo, "Development of sample-scanning electron holography," Microscopy and Microanalysis, vol. 17, supplement 2, pp. 1230-1231, 2011.

[16] H. Lichte, D. Geiger, A. Harscher et al., "Artefacts in electron holography," Ultramicroscopy, vol. 64, no. 1-4, pp. 67-77, 1996. 

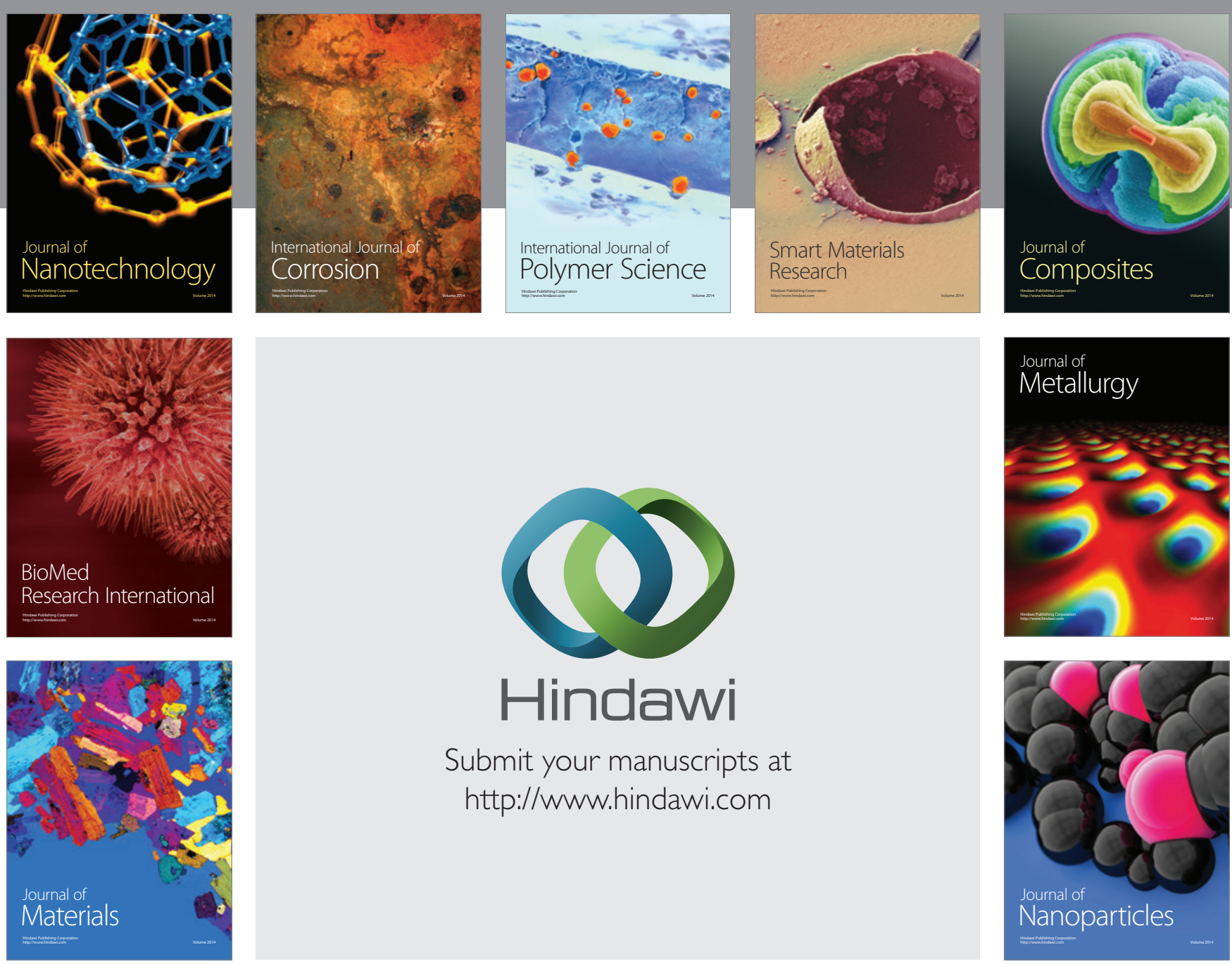

Submit your manuscripts at http://www.hindawi.com
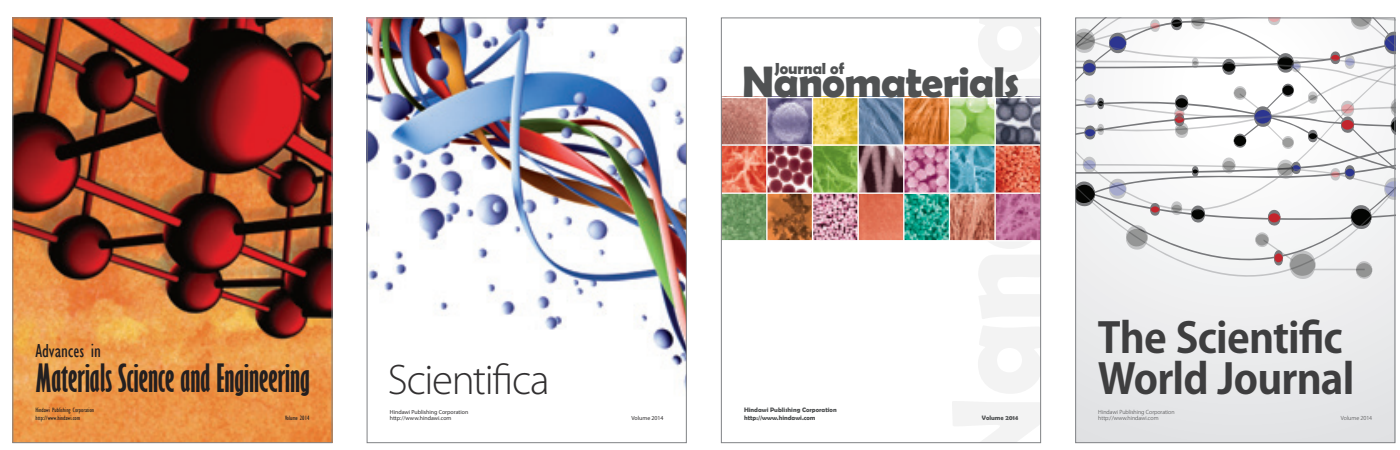

\section{The Scientific World Journal}
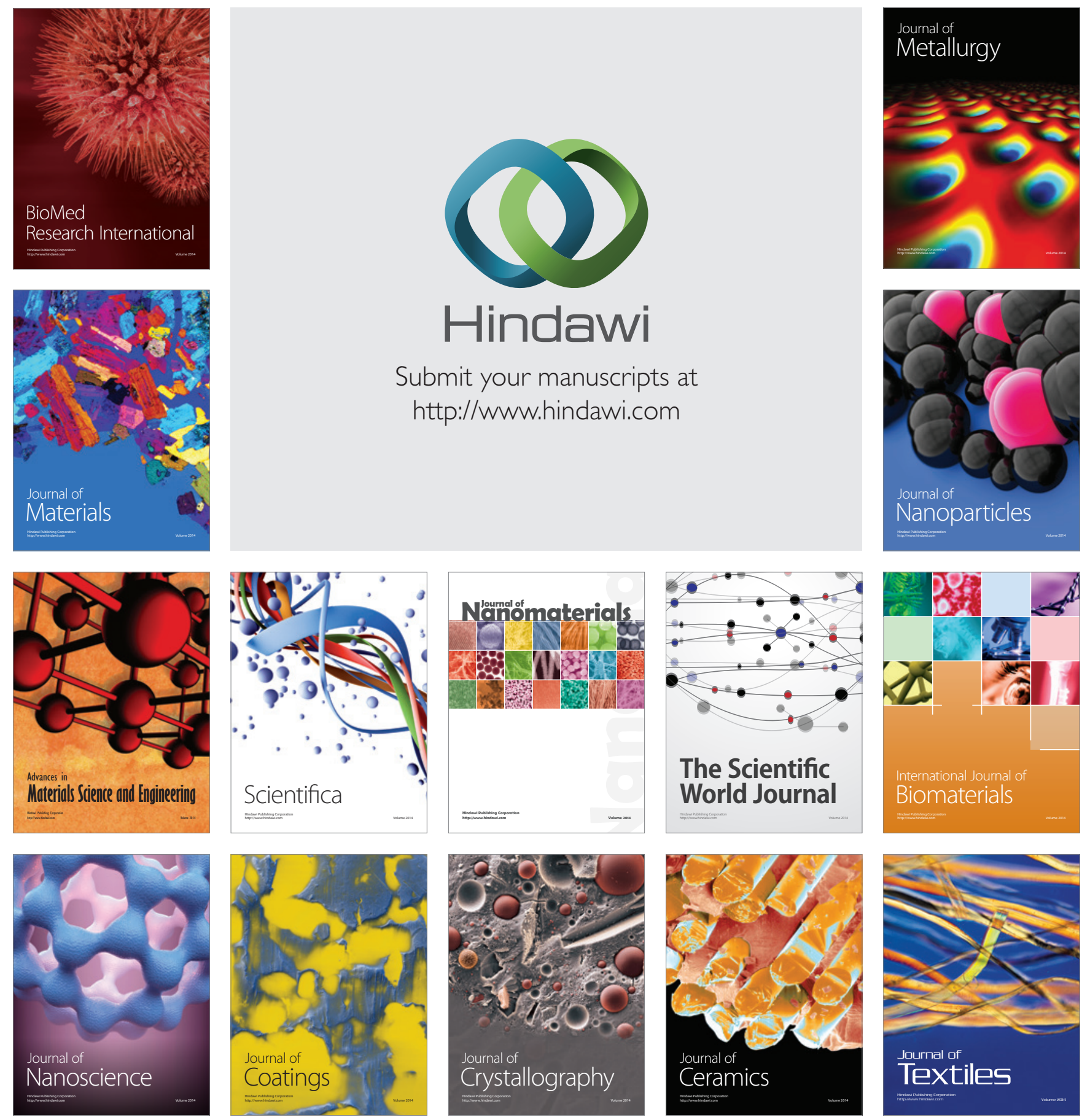